\title{
Symbol Error Probability of Distributed-Alamouti Scheme in Wireless Relay Networks
}

\author{
Trung Q. Duong \\ Radio Communications Group, \\ Blekinge Institute of Technology, SE-372 25, Ronneby, Sweden, \\ e-mail: quang.trung.duong@bth.se
}

\author{
Dac-Binh Ha, Hoai-An Tran, Nguyen-Son Vo \\ Faculty of Electrical and Electronics Engineering, \\ Ho Chi Minh City University of Transport, Vietnam
}

\begin{abstract}
In this paper, we analyze the maximum likelihood decoding performance of non-regenerative cooperation employing Alamouti scheme. Specifically, we derive two closed-form expressions for average symbol error probability (SEP) when the relays are located near by the source or destination. The analytical results are obtained as a single integral with finite limits and an integrand composed solely of trigonometric functions. Assessing the asymptotic (high signal-to-noise ratio) behavior of SEP formulas, we show that the distributed-Alamouti codes achieves a full diversity order. We also perform Monte-Carlo simulations to validate the analysis.
\end{abstract}

Index Terms-Alamouti, distributed space-time codes (DSTCs), non-regenerative relays, symbol error probability (SEP).

\section{INTRODUCTION}

\section{A. Distributed-Alamouti Space-Time Coding Scheme}

Alamouti scheme [1] has been considered as the only orthogonal space-time block code that can provide full rate and full diversity over complex constellation with symbolwise maximum likelihood (ML) decoding complexity. Recently, there exists an extension of Alamouti scheme into cooperative/relay systems where relays simultaneously receive a noisy signal from the source and construct Alamouti spacetime codes in a distributed fashion before relaying the signals to the destination [2]-[6].

\section{B. Related Work}

Distributed-Alamouti scheme dated back to the work in [2], [3], where two single-antenna relays assisted the sourcedestination communication by forming the Alamouti spacetime block code in relay networks. The authors conjectured a diversity order of distributed-Alamouti scheme around one from their simulation. In [5], the average bit error rate (BER) was shown to be proportional to $\log (\mathrm{SNR}) / \mathrm{SNR}^{2}$.

In [4], distributed-Alamouti system was created by using only one single-antenna relay in Protocol III, i.e., the relay and source construct a distributed-Alamouti space-time code and each terminal transmits each row of Alamouti code. Recently, exact closed-form expressions for pairwise error probability of this scheme has been analyzed in [6] where it has been shown that a full diversity order is achieved.

\footnotetext{
${ }^{0}$ This research was supported in part by Consultant Application of Science Technology of Transport Company (CAST) - Vietnam.
}

More recently, we generalized the construction of distributed space-time codes using amicable orthogonal design in relay networks [7]. It has been showed that our scheme can achieve full diversity order and single-symbol ML decoding complexity with the sacrifice of bandwidth efficiency.

\section{Motivation and Results}

Unlike most of previous works [2], [3], [5], [8], which focused on the average bit error rate (BER) of distributedAlamouti space-time code for binary phase-shift keying (BPSK) modulation, we analyze the average symbol error probability (SEP) for $M$-ary phase-shift keying ( $M$-PSK) by using the moment generating function (MGF) method [9], [10]. More specifically, we derive two closed-form expressions of average SEP for $M$-PSK modulation when the relays are close to either the destination or source. Since the asymptotic behavior of the MGF at large signal-to-noise (SNR) reveals a high-SNR slope of the SEP curve [9], [11], we show that distributed-Alamouti scheme achieves full diversity, i.e., a diversity order of two.

\section{Organization and Notations}

The paper is organized as follows. In the following section, we briefly review the cooperative system of two nonregenerative relays based on Alamouti scheme. We then derive two exact closed-form expressions of average SEP and diversity orders when two relays are closely located to both ends in Section III and Section IV, respectively. We show that two average SEP curves agree exactly with the Monte-Carlo simulation in Section V. Section VI provides the conclusion and future work.

Notation: Throughout the paper, we shall use the following notations. Vector is written as bold lower case letter and matrix is written as bold upper case letter. The superscripts $*$ and $\dagger$ stand for the complex conjugate and transpose conjugate. $\boldsymbol{I}_{n}$ represents the $n \times n$ identity matrix. $\|\boldsymbol{A}\|_{\mathrm{F}}$ denotes Frobenius norm of the matrix $A$ and $|x|$ indicates the envelope of $x . \mathbb{E}_{x}\{$.$\} is the expectation operator over the random$ variable $x$. A complex Gaussian distribution with mean $\mu$ and variance $\sigma^{2}$ is denoted by $\mathcal{C N}\left(\mu, \sigma^{2}\right)$. log is the natural logarithm. $\Gamma(a, x)$ is the incomplete gamma function defined as $\Gamma(a, x)=\int_{x}^{\infty} t^{a-1} e^{-t} d t$ and $\mathcal{K}_{0}($.$) is the zeroth-order$ modified Bessel function of the second kind. 


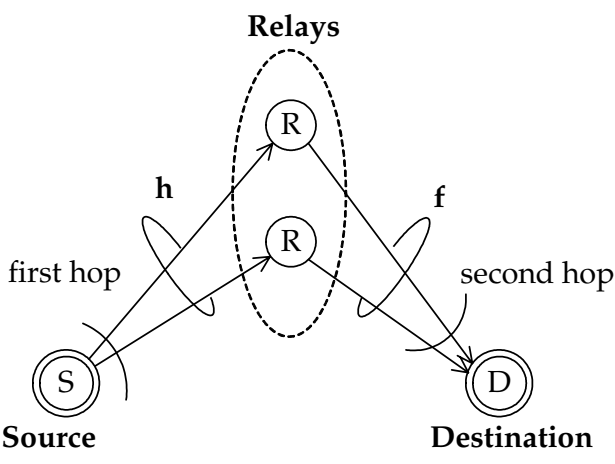

Fig. 1. Dual-hop non-regenerative relay channels.

\section{SYSTEM MODEL}

We consider a dual-hop relay channel, as shown in Fig. 1, where the channel remains constant for $T_{\text {coh }}$ coherence time (an integer multiple of the dual-hop interval) and changes independently to a new value for each coherence time. All terminals are in half-duplex mode and, hence, transmission occurs over two time slots, each with the interval of two symbol periods.

In the first time slot, the source transmits two symbols $s=$ [ $\left.\begin{array}{ll}s_{1} & s_{2}\end{array}\right]$, selected from $M$-PSK signal constellation $\mathcal{S}$, with average transmit power per symbol $\mathcal{P}_{\mathrm{s}}$. During the first hop the received signals $\boldsymbol{y}_{i}=\left[\begin{array}{ll}y_{i}(1) & y_{i}(2)\end{array}\right]$ at $i$ th relay is given by

$$
\boldsymbol{y}_{i}=h_{i} \boldsymbol{s}+\boldsymbol{n}_{\mathrm{R}_{i}}
$$

where $y_{i}(j)$ is the $j$ th symbol received at the $i$ th relay, $h_{i} \sim$ $\mathcal{C N}\left(0, \Omega_{\mathrm{h}}\right)$ is the Rayleigh-fading channel coefficient for the source- $i$ th relay link, $\boldsymbol{n}_{\mathrm{R}_{i}}$ is complex additive white Gaussian noise (AWGN) of variance $N_{0}$, and $i=1,2$.

During the second time slot, the two relays construct Alamouti space-time scheme from the two received signals, and then retransmit a scaled version to the destination with the same power constraint as in the first hop, whereas the source remains silent. To simplify relaying operation, the relaying gain is determined only to satisfy the average power constraint with statistical channel state information (CSI) on $\boldsymbol{h}$ (not its realizations) at the relay. With this semi-blind relaying, the output signal of the two relays are defined as

$$
\boldsymbol{X}=\left[\begin{array}{cc}
x_{1}(1) & x_{2}(1) \\
x_{1}(2) & x_{2}(2)
\end{array}\right]=G\left[\begin{array}{cc}
y_{1}(1) & -y_{2}^{*}(2) \\
y_{1}(2) & y_{2}^{*}(1)
\end{array}\right]
$$

where $x_{i}(j)$ is the $j$ th symbol transmitted from the $i$ th relay and $G$ is the scalar relaying gain defined in the following. The received signal at the destination can be described as

$$
r(j)=\sum_{i=1}^{2} f_{i} x_{i}(j)+n_{D}(j)
$$

where $f_{i} \sim \mathcal{C N}\left(0, \Omega_{\mathrm{f}}\right)$ is the Rayleigh-fading channel coefficient for the $i$ th relay-destination link and $n_{D}(j)$ is complex additive white Gaussian noise (AWGN) of variance $N_{0}$. Note that all the random variable $h_{i}$ and $f_{i}, i=1,2$ are statistically independent and the variations in $\Omega_{\mathrm{A}}, \mathrm{A} \in\{h, f\}$ capture the effect of distance-related path loss in each link. To constrain transmit power at the relay, we have

$$
\mathbb{E}\left\{\|\boldsymbol{X}\|_{\mathrm{F}}^{2}\right\}=4 G^{2}\left(\Omega_{\mathrm{h}} \mathcal{P}_{\mathrm{s}}+N_{0}\right)=\mathbb{E}\left\{\|\boldsymbol{s}\|_{\mathrm{F}}^{2}\right\}
$$

yielding

$$
G^{2}=\frac{1}{2}\left(\Omega_{\mathrm{h}}+\frac{1}{\mathrm{SNR}}\right)^{-1}
$$

where SNR $=\frac{\mathcal{P}_{\mathrm{s}}}{N_{0}}$ is the common SNR of each link without fading [12]. The receive signals at the destination in (3) now can be equivalently described in the matrix form as

$$
r=H s+n
$$

where

$$
\begin{gathered}
\boldsymbol{r}=\left[\begin{array}{c}
r(1) \\
r^{*}(2)
\end{array}\right], \\
\boldsymbol{H}=G\left[\begin{array}{cc}
h_{1} f_{1} & -h_{2}^{*} f_{2} \\
h_{2} f_{2}^{*} & h_{1}^{*} f_{1}^{*}
\end{array}\right],
\end{gathered}
$$

which is complex orthogonal, i.e.,

$$
\boldsymbol{H}^{\dagger} \boldsymbol{H}=G^{2} \sum_{i=1}^{2}\left|h_{i}\right|^{2}\left|f_{i}\right|^{2} \boldsymbol{I}_{2}
$$

and

$$
\boldsymbol{n}=G\left[\begin{array}{cc}
f_{1} n_{\mathrm{R}_{1}}(1) & -f_{2} n_{\mathrm{R}_{2}}^{*}(2) \\
f_{1}^{*} n_{\mathrm{R}_{1}}^{*}(2) & f_{2}^{*} n_{\mathrm{R}_{2}}(1)
\end{array}\right]+\left[\begin{array}{c}
n_{D}(1) \\
n_{D}^{*}(2)
\end{array}\right]
$$

which is zero-mean AWGN of the variance $N_{0}\left(G^{2} \sum_{i=1}^{2}\left|f_{i}\right|^{2}+1\right)$. It is easy to see that the ML decoding of the symbol vector $s$ turns out very simple as two symbols in $s$ are independently decomposed from one another. Therefore, the instantaneous receive SNR is readily written as

$$
\gamma=\operatorname{SNR} \frac{G^{2} \sum_{i=1}^{2}\left|h_{i}\right|^{2}\left|f_{i}\right|^{2}}{G^{2} \sum_{i=1}^{2}\left|f_{i}\right|^{2}+1}
$$

To examine the statistical characteristic of $\gamma$ given in (7) we consider two special cases. If the relays are much closer to the destination than the source, then we may have $G^{2} \sum_{i=1}^{2}\left|f_{i}\right|^{2} \gg$ 1. In this special case, we express $\gamma$ in the following

$$
\gamma=\operatorname{SNR} \frac{\sum_{i=1}^{2}\left|h_{i}\right|^{2}\left|f_{i}\right|^{2}}{\sum_{i=1}^{2}\left|f_{i}\right|^{2}}
$$

On the other hand, if the relays are near the source, then the following expression may hold $G^{2} \sum_{i=1}^{2}\left|f_{i}\right|^{2} \ll 1$. Therefore, the instantaneous receive $\operatorname{SNR} \gamma$ is as follows

$$
\gamma=\operatorname{SNR} G^{2} \sum_{i=1}^{2}\left|h_{i}\right|^{2}\left|f_{i}\right|^{2}
$$




\section{Closed-Form Expression of The Average SEP AND DIVERSITY ORDER}

In this section, on account of the statistical behavior of the instantaneous receive SNR for two special cases as shown in previous section, we now derive the close-form expression of the average SEP and then deduce the diversity order of distributed-Alamouti scheme in such cases.

\section{A. When the relays are close to the destination}

With the instantaneous receive SNR in (8), the MGF of $\gamma$, averaged over channel coefficients, is given by

$$
\phi_{\gamma}(\nu) \triangleq \mathbb{E}_{\gamma}\{\exp (-\nu \gamma)\}=\mathbb{E}_{h_{i}, f_{i}}\{\exp (-\nu \gamma)\}
$$

Since $\mathrm{A}_{i} \sim \mathcal{C N}\left(0, \Omega_{\mathrm{A}}\right), \mathrm{A} \in\{h, f\}$ and $i=1,2$, it is obvious that $\left|\mathrm{A}_{i}\right|^{2}$ obeys an exponential distribution with hazard rate $1 / \Omega_{\mathrm{A}}$. The probability density function (p.d.f.) of $\left|\mathrm{A}_{i}\right|^{2}$ can be written as

$$
p_{\left|\mathrm{A}_{i}\right|^{2}}(x)=\frac{1}{\Omega_{\mathrm{A}}} \exp \left(-x / \Omega_{\mathrm{A}}\right)
$$

Since $h_{i}$ and $f_{i}$ are statistically independent with each other, the MGF of $\gamma$ in (10) can be written as

$$
\begin{aligned}
\phi_{\gamma}(\nu) & =\mathbb{E}_{f_{i}}\left\{\mathbb{E}_{h_{i}}\{\exp (-\nu \gamma)\}\right\} \\
& =\mathbb{E}_{f_{i}}\left\{\prod_{i=1}^{2}\left(1+\operatorname{SNR} \nu \frac{\left|f_{i}\right|^{2}}{\left|f_{1}\right|^{2}+\left|f_{2}\right|^{2}}\right)^{-1}\right\} \\
& =\mathbb{E}_{z}\left\{\left(1+\xi \frac{z}{z+1}\right)^{-1}\left(1+\xi \frac{1}{z+1}\right)^{-1}\right\} \\
& \stackrel{(a)}{=} \int_{0}^{\infty}[(1+(1+\xi) z)(1+\xi+z)]^{-1} d z \\
& =\frac{2 \log (1+\xi)}{\xi(2+\xi)}
\end{aligned}
$$

where $z=\frac{\left|f_{1}\right|^{2}}{\left|f_{2}\right|^{2}}, \xi=\Omega_{\mathrm{h}} \mathrm{SNR} \nu$, and $(a)$ follows immediately from Theorem 2 in Appendix. Using the well-known MGF approach [10], [13] along with (12), we obtain the average SEP of the distributed-Alamouti scheme with $M$-PSK in relay channels as ${ }^{1}$

$$
\begin{aligned}
P_{\mathrm{e}} & =\frac{1}{\pi} \int_{0}^{\pi-\frac{\pi}{M}} \phi_{\gamma}\left(\frac{g_{\mathrm{MPSK}}}{\sin ^{2} \theta}\right) d \theta \\
& =\frac{1}{\pi} \int_{0}^{\pi-\frac{\pi}{M}} \frac{2 \sin ^{4} \theta \log \left(1+\frac{\psi}{\sin ^{2} \theta}\right)}{\psi\left(2 \sin ^{2} \theta+\psi\right)} d \theta
\end{aligned}
$$

where $\psi=\Omega_{\mathrm{h}} \mathrm{SNR} g_{\mathrm{MPSK}}$ and $g_{\mathrm{MPSK}}=\sin ^{2}(\pi / M)$.

\section{B. When the relays are close to the source}

In this section, we will consider the case when the two relays are close to the source. Following the same steps as in

\footnotetext{
${ }^{1}$ The result can be applied to other binary and $M$-ary signals in a straightforward way (see, e.g., [13]).
}

Section III-A and from (9), the MGF of $\gamma$ can be described as

$$
\begin{aligned}
& \phi_{\gamma}(\nu)=\mathbb{E}_{h_{i}, f_{i}}\left\{\prod_{i=1}^{2} \exp \left(-G^{2} \operatorname{SNR} \nu\left|h_{i}\right|^{2}\left|f_{i}\right|^{2}\right)\right\} \\
& =\left[\int_{0}^{\infty} \exp \left(-G^{2} \operatorname{SNR} \nu t\right) p_{T}(t) d t\right]^{2} \\
& =\left[\int_{0}^{\infty} \frac{2}{\Omega} \exp \left(-G^{2} \mathrm{SNR} \nu t\right) \mathcal{K}_{0}\left(2 \sqrt{\frac{t}{\Omega}}\right) d t\right]^{2} \\
& =[\lambda \exp (\lambda) \Gamma(0, \lambda)]^{2}
\end{aligned}
$$

where $T=\left|h_{i}\right|^{2}\left|f_{i}\right|^{2}, \Omega=\Omega_{\mathrm{h}} \Omega_{\mathrm{f}}, \lambda=\left(G^{2} \mathrm{SNR} \nu \Omega\right)^{-1},(16)$ follows immediately from Theorem 3 in Appendix, and (17) can be obtained from the change of variable $v=G^{2} \mathrm{SNR} \nu t$ along with [14, eq. (8.353.4)]. Therefore, similarly as in the previous subsection, the SEP is given by

$$
P_{\mathrm{e}}=\frac{1}{\pi} \int_{0}^{\pi-\frac{\pi}{M}}\left[\tau \sin ^{2} \theta \exp \left(\tau \sin ^{2} \theta\right) \Gamma\left(0, \tau \sin ^{2} \theta\right)\right]^{2} d \theta
$$

where $\tau=\left(G^{2} \mathrm{SNR} \Omega g_{\mathrm{MPSK}}\right)^{-1}$.

\section{High-SNR Characteristic: Diversity Order}

We now assess the effect of cooperative diversity on the SEP behavior in a high-SNR regime. The diversity impact of non-regenerative cooperation on a high-SNR slope of the SEP curve can be quantified by the following theorem.

Theorem 1 (Achievable Diversity Order): The nonregenerative cooperation of distributed-Alamouti scheme provides maximum diversity order, i.e.,

$$
D \triangleq \lim _{\mathrm{SNR} \rightarrow \infty} \frac{-\log P_{\mathrm{e}}}{\log (\mathrm{SNR})}=2 .
$$

Proof: See Appendix C

\section{Numerical Results}

In this section, we validate our analysis by comparing with Monte-Carlo simulation. In the following numerical examples, we consider the non-regenerative relay protocol employing Alamouti code as in Section II. We assume collinear geometry for locations of three communicating terminals, as shown in Fig. 2. The path-loss of each link follows an exponential-decay model: if the distance between the source and destination is equal to $d$, then $\Omega_{0} \propto d^{-\alpha}$ where the exponent $\alpha=4$ corresponding to a typical non line-of-sight propagation. Then, $\Omega_{\mathrm{h}}=\epsilon^{-\alpha} \Omega_{0}$ and $\Omega_{\mathrm{f}}=(1-\epsilon)^{-\alpha} \Omega_{0}$.

Fig. 3 and Fig. 4 show the SEP of QPSK versus SNR when the relay approaches the destination with $\epsilon=0.7$ and $\epsilon=0.8$ and the source with $\epsilon=0.2$ and $\epsilon=0.3$, respectively. As seen from both figures, analytical and simulated SEP curves match exactly. Observe that the PEP slops for $\epsilon=0.7$ and $\epsilon=0.8$ are identical at the high SNR regime, as speculated in Theorem 1. The same observation can be obtained for $\epsilon=0.2$ and $\epsilon=0.3$. For comparison, two examples demonstrate the 
performance is decreased when the relay approaches both end, e.g., the SEP for $\epsilon=0.3$ is slightly less than that for $\epsilon=0.2$ and a 3dB-gain in SEP performance can be obtained with $\epsilon=0.7$ compared to the case with $\epsilon=0.8$.

\section{APPENDIX}

\section{A. Auxiliary Results}

The following lemmas will be useful in the paper

Lemma 1: Let $a>0$ be a finite constant and

$$
f(x)=\frac{2 \log (1+a x)}{a x(2+a x)}, \quad x>0
$$

We have

$$
f_{x \uparrow} \triangleq \lim _{x \rightarrow \infty} \frac{-\log f(x)}{\log x}=2
$$

Proof: It follows immediately from (20) and (21) that

$$
\begin{aligned}
& f_{x \uparrow}=\underbrace{\lim _{x \rightarrow \infty} \frac{-\log \log (1+a x)}{\log x}}_{\stackrel{(b)}{=} 0}+\underbrace{\lim _{x \rightarrow \infty} \frac{\log (a x)}{\log x}}_{\stackrel{(c)}{=} 1} \\
& +\underbrace{\lim _{x \rightarrow \infty} \frac{\log \left(1+\frac{a x}{2}\right)}{\log x}}_{\stackrel{(d)}{=} 1}=2
\end{aligned}
$$

where $(b),(c)$, and $(d)$ follow immediately by applying l'Hôspital rule.

Lemma 2: Let

$$
g(\zeta)=[\zeta \exp (\zeta) \Gamma(0, \zeta)]^{2}, \quad \zeta>0
$$

Let $\zeta=\alpha \frac{\beta x+1}{x^{2}}$ and $\alpha, \beta>0$ be finite constants. We have

$$
g_{x \uparrow} \triangleq \lim _{x \rightarrow \infty} \frac{-\log g(x)}{\log x}=2
$$

Proof: Substituting $\zeta=\alpha \frac{\beta x+1}{x^{2}}$ into (23), it follows immediately from (24) that

$$
\begin{aligned}
& g_{x \uparrow}=-2\left[\lim _{x \rightarrow \infty} \frac{-\log \left(\frac{\beta x+1}{x^{2}}\right)}{\log x}+\lim _{x \rightarrow \infty} \frac{\beta x+1}{x^{2} \log x}\right. \\
& \left.+\lim _{x \rightarrow \infty} \frac{\Gamma\left(0, \alpha \frac{\beta x+1}{x^{2}}\right)}{\log x}\right]=-2[-2+\underbrace{\lim _{x \rightarrow \infty} \frac{\log (1+\beta x)}{\log x}}_{\stackrel{(e)}{=} 1} \\
& +\underbrace{\lim _{x \rightarrow \infty} \frac{\beta x+1}{x^{2} \log x}}_{\underline{\underline{(f)} 0} 0}+\underbrace{\lim _{x \rightarrow \infty} \frac{\Gamma\left(0, \alpha \frac{\beta x+1}{x^{2}}\right)}{\log x}}_{\underline{\underline{g}} 0}]=2
\end{aligned}
$$

where $(e),(f)$ follow immediately by l'Hôspital rule and $(g)$ follows from the Laguerre $^{2}$ polynomial series representation of incomplete gamma function [14] together with l'Hôspital rule .

\footnotetext{
${ }^{2}$ The incomplete gamma function can be described as $\Gamma(0, x)=$ $e^{-x} \sum_{n=0}^{\infty} \frac{L_{n}(x)}{n+1}$ where $L_{n}(x)=\sum_{m=0}^{n}(-1)^{m}\left(n ! x^{m}\right) /(m !(n-m) ! m !)$ is the Laguerre polynomial of order $n$.
}

\section{B. A Ratio and Product Distribution}

Theorem 2 (Ratio Distribution): Let

$$
\begin{aligned}
& X \sim \Upsilon(1 / \Omega) \\
& Y \sim \Upsilon(1 / \Omega)
\end{aligned}
$$

be statistically independent and identically distributed (i.i.d.) exponential r.v.'s. Suppose the ratio $Z$ of the form

$$
Z=\frac{X}{Y}
$$

Then, we obtain the p.d.f. of random variable $Z$ as

$$
p_{Z}(z)=(z+1)^{-2}
$$

Proof: Note that

$$
\begin{aligned}
p_{Z}(z) & =\int_{0}^{\infty} y p_{X Y}(y z, y) d y \\
& =\int_{0}^{\infty} \frac{y}{\Omega^{2}} \exp \left(-y \frac{z+1}{\Omega}\right) d y \\
& =(z+1)^{-2}
\end{aligned}
$$

\section{Theorem 3 (Product Distribution): Let}

$$
\begin{aligned}
& X \sim \Upsilon\left(1 / \Omega_{x}\right) \\
& Y \sim \Upsilon\left(1 / \Omega_{y}\right)
\end{aligned}
$$

be statistically independent and not necessarily identically distributed (i.n.i.d.) exponential r.v.'s. Suppose the product $T$ of the form

$$
T=X Y
$$

Then, we have

$$
p_{T}(t)=\frac{2}{\Omega_{x} \Omega_{y}} \mathcal{K}_{0}\left(2 \sqrt{\frac{t}{\Omega_{x} \Omega_{y}}}\right)
$$

where $\mathcal{K}_{0}($.$) is the zeroth-order modified Bessel function of$ the second kind.

Proof: Note that

$$
\begin{aligned}
F_{T}(t) & =\operatorname{Pr}\{X Y \leq t\} \\
& =\mathbb{E}_{X}\left\{F_{T \mid X}(t)\right\} \\
& =\mathbb{E}_{X}\left\{1-\exp \left[-\frac{t}{x \Omega_{y}}\right]\right\} \\
& =1-\frac{1}{\Omega_{x}} \int_{0}^{\infty} \exp \left[-\frac{t}{x \Omega_{y}}-\frac{x}{\Omega_{x}}\right] d x
\end{aligned}
$$

The p.d.f. of $T$ follows immediately from differentiating (31) with respect to $t$.

$$
\begin{aligned}
p_{T}(t) & =\frac{1}{\Omega_{x} \Omega_{y}} \int_{0}^{\infty} \frac{1}{x} \exp \left[-\frac{t}{x \Omega_{y}}-\frac{x}{\Omega_{x}}\right] d x \\
& =\frac{2}{\Omega_{x} \Omega_{y}} \mathcal{K}_{0}\left(2 \sqrt{\frac{t}{\Omega_{x} \Omega_{y}}}\right)
\end{aligned}
$$

where the last equality follows from the change of variable $u=\frac{x}{\Omega_{x}}$ along with [14, eq. (8.432.6)] as desired. 


\section{Proof of Theorem 1}

Since the asymptotic behavior of the MGF $\phi_{\gamma}(\nu)$ at large SNR reveals a high-SNR slope of the SEP curve, we have [11]

$$
D=\lim _{\mathrm{SNR} \rightarrow \infty} \frac{-\log \phi_{\gamma}\left(g_{\mathrm{MPSK}}\right)}{\log (\mathrm{SNR})} .
$$

Hence, it follow immediately from (12) and Lemma 1 with $a=\Omega_{\mathrm{h}} g_{\mathrm{MPSK}}$ that $D=2$ when the relays are close to the destination. Also, from (17) and Lemma 2 with $\alpha=\frac{2}{\Omega_{\mathrm{h}} \Omega_{\mathrm{f}} g_{\mathrm{MPSK}}}$ and $\beta=\Omega_{\mathrm{h}}$, we can obtain $D=2$ as in (19) when the relays are much close to the source.

\section{REFERENCES}

[1] S. M. Alamouti, "A simple transmit diversity technique for wireless communications," IEEE J. Sel. Areas Commun., vol. 16, no. 8, pp. 14511458, Oct. 1998.

[2] Y. Hua, Y. Mei, and Y. Chang, "Wireless antennas - making wireless communications perform like wireline communications," in IEEE AP-S Topical Conference on Wireless Communication Technology, Honoluu, Hawaii, Oct. 2003, pp. 1-27, invited paper.

[3] _ , "Parallel wireless mobile relays with space time modulations," in IEEE Workshop on Statistical Signal Processing, St. Louis, Missouri, Oct. 2003, pp. 375-378.

[4] R. U. Nabar, H. Bölcskei, and F. W. Kneubühler, "Fading relay channels: Performance limits and space-time signal design," IEEE J. Sel. Areas Commun., vol. 22, no. 8, pp. 1099-1109, Aug. 2004.

[5] Y. Chang and Y. Hua, "Diversity analysis of orthogonal space-time modulation for distributed wireless relays," in IEEE Intl. Conf. on Acoustics, Speech, and Signal Processing, Montreal, Canada, May 2004, pp. 561-564.

[6] T. Q. Duong, V.-T. Nguyen, and V.-K. Nguyen, "Exact pairwise error probability of distributed space-time coding in wireless relays networks," in IEEE 7th International Symposium on Communications and Information Technologies, Sydney, Australia, Oct. 2007.

[7] T. Q. Duong and H.-A. Tran, "Distributed space-time block codes with amicable orthogonal designs," in IEEE Radio and Wireless Symposium, Florida, USA, Jan. 2008.

[8] P. A. Anghel and M. Kaveh, "On the performance of distributed spacetime coding system with one and two non-regenerative relays," IEEE Trans. Wireless Commun., vol. 5, no. 3, pp. 682-691, Mar. 2006.

[9] M. K. Simon and M.-S. Alouini, "A unified approach to the performance analysis of digital communication over generalized fading channels," Proc. IEEE, vol. 86, no. 9, pp. 1860-1877, Sep. 1998.

[10] — Digital Communication over Fading Channels: A Unified Approach to Performance Analysis. New York: Wiley, 2000.

[11] H. Shin, T. Q. Duong, and E. K. Hong, "MIMO cooperative diversity with amplify-and-forward relaying," IEEE Trans. Commun., Jul. 2007, accepted pending revision.

[12] J. N. Laneman, D. N. C. Tse, and G. W. Wornell, "Cooperative diversity in wireless networks: Efficient protocols and outage behavior," IEEE Trans. Inf. Theory, vol. 50, no. 10, pp. 3062-3080, Dec. 2004.

[13] M. K. Simon and M.-S. Alouini, "A unified approach to the performance analysis of digital communication over generalized fading channels," Proc. IEEE, vol. 86, no. 9, pp. 1860-1877, Sep. 1998.

[14] I. S. Gradshteyn and I. M. Ryzhik, Table of Integrals, Series, and Products, 6th ed. San Diego, CA: Academic, 2000.

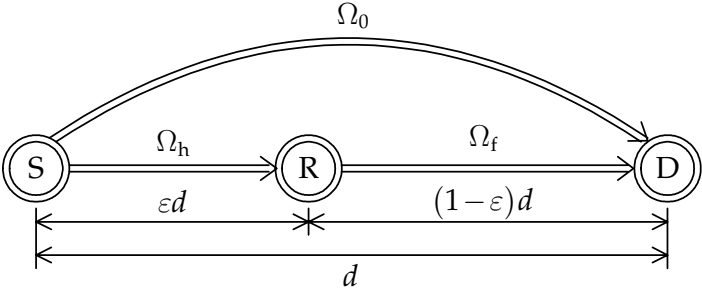

Fig. 2. Collinear topology with an exponential-decay path loss model where $\Omega_{0} \propto d^{-\alpha}, \Omega_{\mathrm{h}}=\varepsilon^{-\alpha} \Omega_{0}$, and $\Omega_{\mathrm{f}}=(1-\varepsilon)^{-\alpha} \Omega_{0}$ with $\alpha=4$.

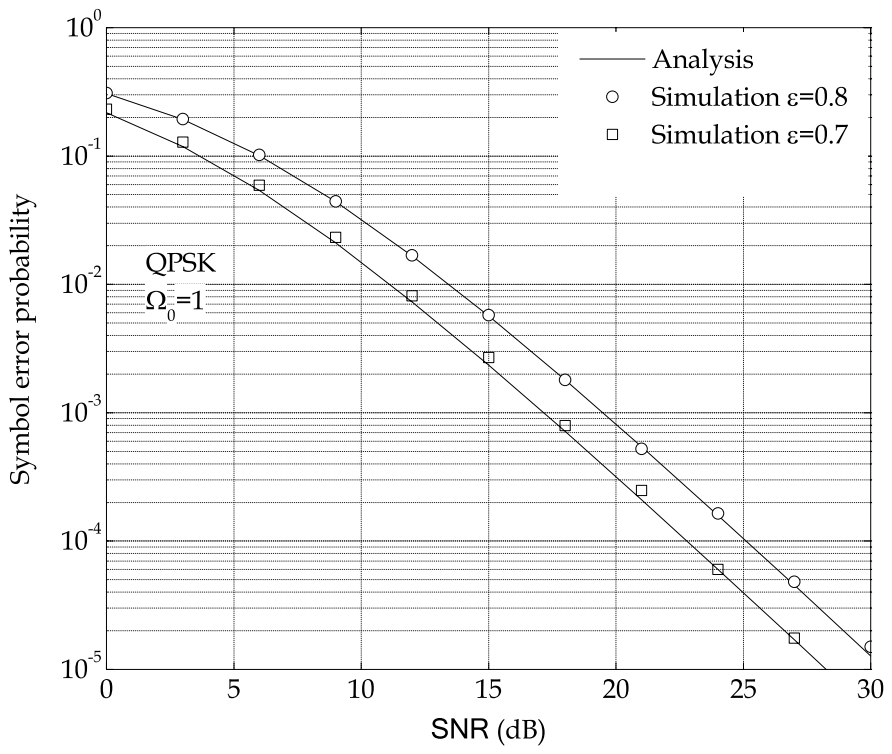

Fig. 3. Symbol error probability of QPSK versus SNR in non-regenerative relay channels employing Alamouti scheme when $\epsilon=0.7$ and $\epsilon=0.8$ (close the destination). $\Omega_{0}=1$.

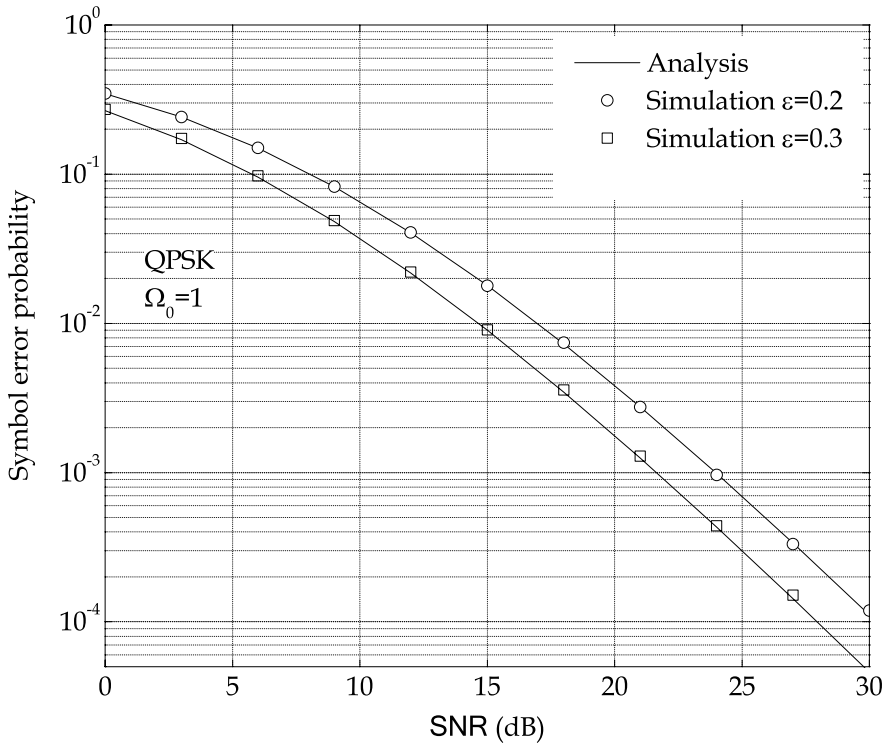

Fig. 4. Symbol error probability of QPSK versus SNR in non-regenerative relay channels employing Alamouti scheme when $\epsilon=0.2$ and $\epsilon=0.3$ (close the source). $\Omega_{0}=1$ 